\title{
Paolo Carile, Huguenots sans frontières. Voyage et écriture à la Renaissance et à l'Âge classique
}

\section{Lionello Sozzi}

\section{(2) OpenEdition}

1 Journals

\section{Edizione digitale}

URL: http://journals.openedition.org/studifrancesi/29887

DOI: $10.4000 /$ studifrancesi.29887

ISSN: 2427-5856

\section{Editore}

Rosenberg \& Sellier

\section{Edizione cartacea}

Data di pubblicazione: 1 avril 2006

Paginazione: 119-120

ISSN: 0039-2944

\section{Notizia bibliografica digitale}

Lionello Sozzi, «Paolo Carile, Huquenots sans frontières. Voyage et écriture à la Renaissance et à l'Âge classique», Studi Francesi [Online], 148 (XLX | I) | 2006, online dal 30 novembre 2015, consultato il 19 avril 2021. URL: http://journals.openedition.org/studifrancesi/29887 ; DOI: https://doi.org/10.4000/ studifrancesi.29887

Questo documento è stato generato automaticamente il 19 avril 2021.

\section{(c)}

Studi Francesi è distribuita con Licenza Creative Commons Attribuzione - Non commerciale - Non opere derivate 4.0 Internazionale. 


\title{
Paolo Carile, Huguenots sans frontières. Voyage et écriture à la Renaissance et à l'Âge classique
}

\author{
Lionello Sozzi
}

\section{NOTIZIA}

Paolo Carile, Huguenots sans frontières. Voyage et écriture à la Renaissance et à l'Âge classique, Paris, Champion, 2001, pp. II-319.

1 Con un ritardo di cui chiediamo venia all'autore e ai lettori segnaliamo questo importante contributo agli studi sulla littérature de voyages: viaggi in mondi esotici, "primitivi", "diversi", lontani, in ogni senso, dall'orizzonte culturale occidentale. Dopo aver trattato questo vasto tema nei volumi Lo sguardo impedito, del 1987, e Écritures de l'ailleurs, del 2000, e dopo aver avviato parallelamente la raccolta di importanti contributi e testimonianze nei due volumi Sur la route des Indes Orientales, rispettivamente del 1995 e del 2002, e in altri due volumi dal titolo Aspects de la francophonie dans l'océan Indien (1996-2000), Paolo Carile ha scelto, in questo nuovo libro, percorsi più infrequentati $\mathrm{e}$, proprio per questo, particolarmente stimolanti e attraenti.

Quel che innanzi tutto colpisce in queste pagine è la ricchezza dei riferimenti bibliografici, l'aggiornamento irreprensibile dei dati libreschi: gli argomenti su cui Carile si intrattiene sono ormai da vari decenni oggetto di assidue ricerche e lo studioso è perfettamente al corrente di quanto si è fatto, si fa e si pubblica nei più vari Paesi e soprattutto nel Canada francofono, secondo il progressivo sviluppo di una produzione storico-critica di cui forse in Europa non si parla a sufficienza. Questo però è solo lo sfondo di ricerche che si muovono, come già si diceva, per vie poco battute, anche se lo studioso ha modelli cui rende il dovuto omaggio nel primo capitolo, da Giuliano Gliozzi a Frank Lestringant ad Alain Beaulieu. I testi e gli autori esplorati non sono dei più noti ma proprio per questo suscitano interesse e riservano sorprese. Si inizia col mito 
insulare di Taprobane e coi progetti di espansione nell'oceano Indiano quali prendono forma in autori portoghesi e poi francesi come ad esempio Jean Parmentier (un autore che ci è caro ritrovare in queste pagine, così legato al dibattito sulla dignitas hominis) o La Popelinière, o altri ugonotti come Jacques Pronis o Henri Duques-ne (cui risale il progetto di una "repubblica" ugonotta alla Réunion) o François Leguat, i cui Voyages et aventures lo studioso considera «une des œuvres littéraires les plus représentatives du calvinisme français du début du XVIII ${ }^{\mathrm{e}}$ siècle» $(\mathrm{p}, 80)$. Affiorano, nelle opere di questi autori, le immagini di isole-simbolo sulle quali «l'imaginaire occidental semble avoir situé, pendant longtemps, les sources primordiales et les archétypes du bonheur et de la désolation» (p. 95). Si tratta, aggiunge Carile, di un ailleurs mythifié che di rado è vera apertura all'altro e al diverso, che può esprimere semplicemente un «désir de fuite», sensazioni effimere e auto-contemplative. Altri capitoli del libro riguardano una figura più nota e di maggior rilievo, quella di Marc Lescarbot, nei cui scritti affiora il desiderio di un «syncrétisme culturel» e di un «renouveau spirituel réalisé en douceur» (p. 167). Altre sezioni del volume trattano il tema affascinante della figura mitica del CafroOttentotto quale è diffusa nella cultura francese dell'età classica, e qui si staglia il profilo avvincente di Guillaume de Laujardière, la cui avventura in Africa è giudicata da Carile «l'une des plus romanesques» tra quelle da lui ripercorse (p. 198). Altre parti riguardano un tema ancora diverso, la cultura francofona alle Antille e l'Histoire naturelle et morale des îles Antilles di Charles de Rochefort, del 1658: un autore la cui identità e il cui «itinéraire mouvementé» (p. 219) lo studioso sa tracciare con sicura erudizione. Chiudono il libro il capitolo sul laboratoire créole e poi quello sull'unico settore che non riguarda il mondo primitivo ma i viaggi in Italia di Nicolas Audebert e Maximilien Misson: capitolo, per altro, che è gradito qui menzionare perché vi si utilizza quell'edizione del Voyage di Audebert che curò a suo tempo uno studioso torinese prematuramente scomparso e sempre rimpianto, Adalberto Olivero.

Malamente riassunto nei termini suddetti, il libro potrà sembrare dispersivo. In realtà, varie filières danno unità a un insieme di saggi in cui l'erudizione non lascia in ombra $\mathrm{i}$ presupposti antropologici e anche, diremmo, etico-politici. Innanzi tutto, infatti, viene in luce, nei testi presi in esame, il persistere di un atteggiamento polemico e negativo nei confronti di primitivi che non si riconducono affatto al profilo abusato del "buon selvaggio" ma che piuttosto coincidono col suo opposto, con quello che Ronald Meek ha chiamato the ignoble savage: visione negativa che è destinata, ovviamente, a fungere da sostegno all'etnocidio e al colonialismo, a una politica di conquista, cioè, di cui lo studioso conosce tutte le fasi e tutte le motivazioni, dimostrando opportunamente come anche la scrittura più "letteraria" vada ancorata a una ben precisa realtà politica e socio-economica. Sostiene il libro, cioè, una. legittima protesta umanitaria cui l'autore non dà esplicito spazio e che tuttavia si coglie in filigrana in tante sue pagine.

In secondo luogo, Carile mette in luce le premesse culturali di "scritture" che non sono mai neutre: viaggiatori, esploratori, missionari, proiettano sull'ailleurs i loro archetipi mentali, le loro convinzioni, le figure del loro imaginaire, vedono i "diversi", cioè, secondo schemi pregiudiziali e deformanti. Tutto ciò non impedisce allo studioso, per altro, di dar rilievo anche ai dati positivi di un percorso storico e di scoperta che si risolve innanzi tutto in quella che chiama la «révolution cosmographique de l'époque» (p. 17), un percorso, per di più, lungo il quale emerge con convincente evidenza la tesi forse centrale del volume, e cioè l'idea che la fede protestante e la specifica identità di autori/viaggiatori legati al credo di Calvino, nomadi, esuli dalla loro patria, convinti di poter creare altrove la "nuova Gerusalemme", rendono quei testimoni, appunto perché 
provenienti da Paesi in cui erano vissuti in condizioni minoritarie, di persecuzione e in ogni caso di estraneità rispetto all'ideologia dominante, particolarmente idonei e intendere l'altro e il diverso nella loro specificità, diversamente dalla strategia cui si adeguavano, ad esempio, Gesuiti, Benedettini o Francescani, sempre ispirata al progetto della forzata evangelizzazione, se non ai criteri dell'esplicito appoggio alla politica coloniale dei governi europei. Lo studioso infatti si chiede «s'il existe chez les calvinistes une manière propre d'envisager et de décrire le rapport à l'altérité anthropologique et géographique des Nouveaux Mondes» (p. 211), ma la risposta in fondo egli l'ha già data quando ha scritto non solo che, trattandosi di viaggiatorimercanti, essi finirono per portare la loro attenzione a vari aspetti della cultura materiale e alle mentalità locali liberandosi almeno in parte dei riflessi mentali tipici dell'Occidente e approdando a un primo oggettivismo etnografico, ma anche «que les calvinistes français, dans leur nomadisme hors des frontières de leur pays, emportaient avec eux une expérience acquise dans leur propre patrie, celle de l'appartenance à une culture minoritaire. Ils étaient donc en mesure d'élaborer des pratiques culturelles de survie», le stesse che consentirono loro di guardare agli "altri" con l'intento di scoprirne la specificità, insomma di «comprendre l'altérité» (pp.195-6). È qui, a nostro avviso, il senso più vero del libro e si tratta, teniamo a dire, di una conclusione del tutto convincente che è formulata non in termini pregiudiziali ma come esito di pazienti ricerche e di molteplici e accattivanti letture. 